\title{
Access Economy for Storage in Energy Communities
}

\author{
Vespermann, Niklas; Hamacher, Thomas; Kazempour, Jalal
}

Published in:

IEEE Transactions on Power Systems

Link to article, DOI:

10.1109/TPWRS.2020.3033999

Publication date:

2021

Document Version

Peer reviewed version

Link back to DTU Orbit

Citation (APA):

Vespermann, N., Hamacher, T., \& Kazempour, J. (2021). Access Economy for Storage in Energy Communities. IEEE Transactions on Power Systems, 36(3), 2234 - 2250. https://doi.org/10.1109/TPWRS.2020.3033999

\section{General rights}

Copyright and moral rights for the publications made accessible in the public portal are retained by the authors and/or other copyright owners and it is a condition of accessing publications that users recognise and abide by the legal requirements associated with these rights.

- Users may download and print one copy of any publication from the public portal for the purpose of private study or research.

- You may not further distribute the material or use it for any profit-making activity or commercial gain

- You may freely distribute the URL identifying the publication in the public portal

If you believe that this document breaches copyright please contact us providing details, and we will remove access to the work immediately and investigate your claim 
The Internet of

Things Meets

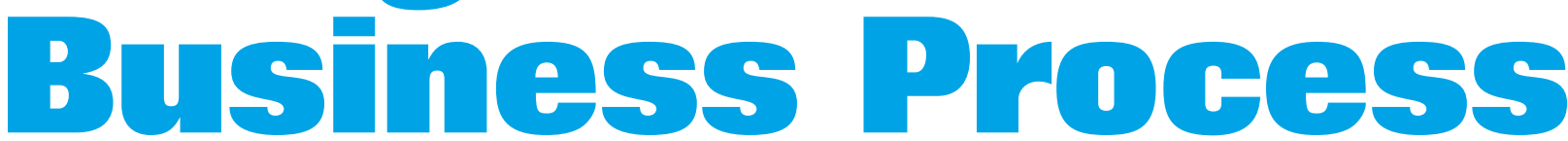

Management Amaniesto

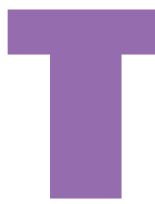

he Internet of Things (IoT) refers to a network of connected devices that collects and exchanges data through the Internet. These things can be artificial or natural and interact as autonomous agents that form a complex system. In turn, business process management (BPM) was established to analyze, discover, design, implement, execute, monitor, and evolve collaborative business processes within and across organizations. While the IoT and BPM have been regarded as separate topics in research and in practice, we strongly believe that, on the one hand, the management of IoT applications will greatly benefit from BPM concepts, methods, and technologies. On the other hand, the IoT poses challenges that will require enhancements and extensions of the current state of the art in the BPM field. In this article, we question the extent to which

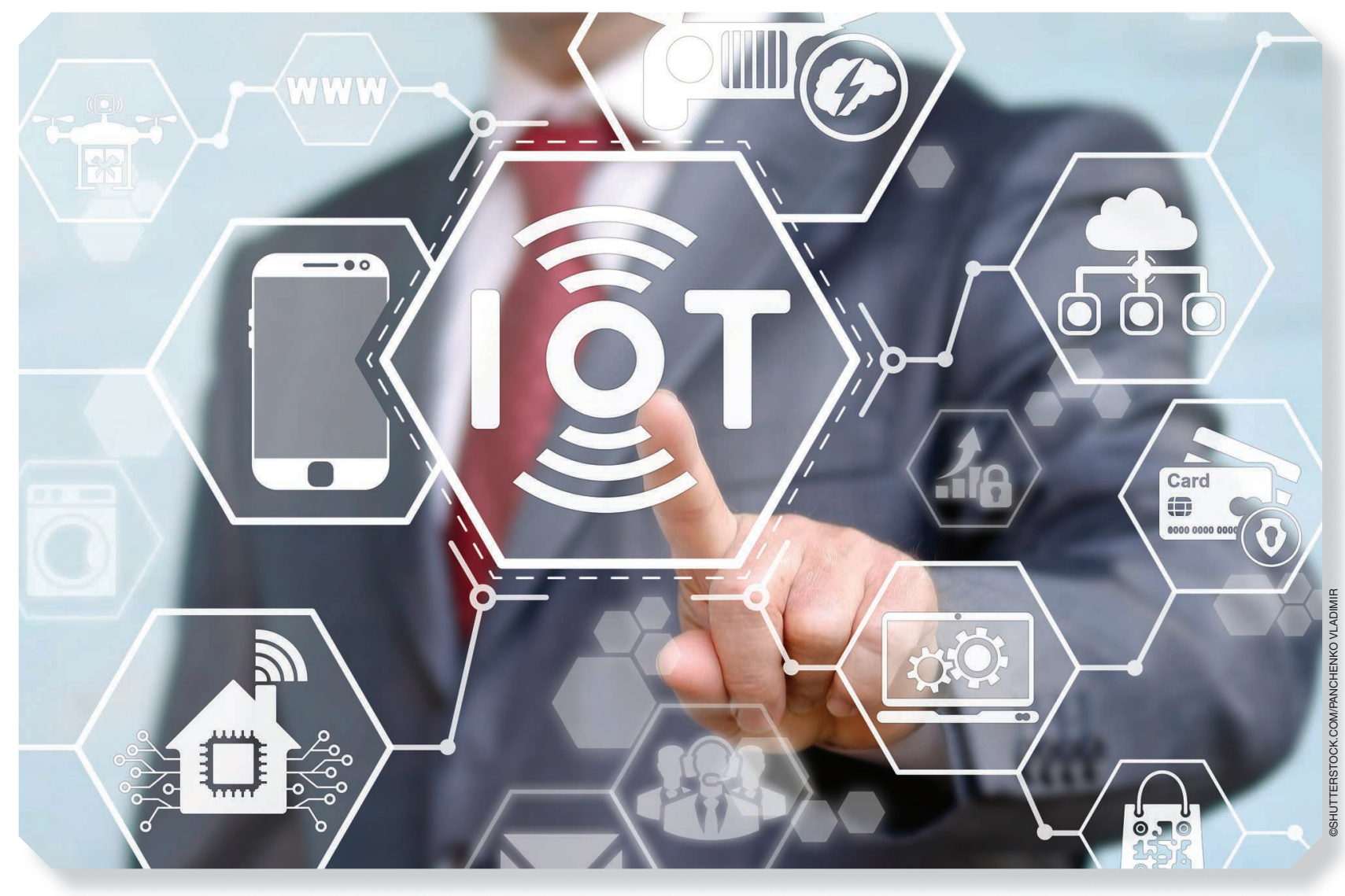

Digital Object Identifier 10.1109/MSMC.2020.3003135

Date of current version: 21 October 2020 
by Christian Janiesch, Agnes Koschmider, Massimo Mecella, Barbara Weber, Andrea Burattin, Claudio Di Ciccio, Giancarlo Fortino, Avigdor Gal, Udo Kannengiesser, Francesco Leotta, Felix Mannhardt, Andrea Marrella, Jan Mendling, Andreas Oberweis, Manfred Reichert, Stefanie Rinderle-Ma, Estefanía Serral, WenZhan Song, Jianwen Su, Victoria Torres, Matthias Weidlich, Mathias Weske, and Liang Zhang

these two paradigms can be combined, and we discuss emerging challenges and intersections from a research and practitioner's point of view in terms of complex software systems development.

\section{Background}

Our world is increasingly linked through a large number of connected devices that typically are embedded in electrical/electronical components and equipped with sensors and actuators that enable sensing, acting on or reacting to, collecting, and exchanging data via various communication networks, including the Internet, thereby forming the IoT (see [24] and "The Internet of Things"). This enables the continuous monitoring of phenomena based on sensing devices (wearables, beacons, smartphones, machine sensors, and so forth), and it creates analytics opportunities in smart environments (smart homes, connected cars, smart logistics, Industry 4.0, and so on) as well as the possibility to actuate feedback. Therefore, the IoT contributes to the recent trend known as big data, and it is one of the three main sources of information beside human-sourced and process-mediated data.

Business processes (see "Business Process Management") represent a specific ordering of tasks and activities across time and places to serve a goal, and they often provide the driving force for system development. Process analytics, execution, and monitoring based on IoT data can enable an even more comprehensive view of systems and realize unused potential for optimization. As an example, during the past, process analytics and, in particular, process mining have been hampered by the fact that procedures are often incomplete and erroneous; with the IoT producing a large amount of data that is stored in the cloud [1], even more information become available for analysis, possibly resolving issues of incompleteness and enabling the provision of error correction methods based on multiple data items [2]. In the literature, some works are emerging on combining BPM and the IoT, e.g., utilizing sensor data to enable the actuation of services [3] and adapting running business processes to continuously align them with the state of the things (e.g., assets, humans, and machines) [4]. Still, there are many open challenges to be tackled. Both BPM and the IoT will benefit from a wider integration.

\section{How Can the IoT Benefit From BPM?}

Let us consider a complex system that includes multiple components interacting within a smart environment and that is aware of the components' locations, movements, and interactions. Such a system can be a smart factory with autonomous robots, a retirement home with connected residents, and, at a larger scale, a smart city. While the parties in the system can track the movements of each component and relate multiple components' behaviors to each other, they do not know the components' agendas. Often, their interactions are based on habits, i.e., routine low-level processes, which represent recurring tasks. Some of these routines are more time and cost critical than others, some may be dangerous or endanger others, and some may just be inefficient or superfluous. Knowing their agendas, goals, and procedures can enable a better basis for planning, execution, and safety.

The solution to typical challenges related to the IoT, such as scalability (a massive number of devices, reliable coverage, and power consumption problems), energy harvesting, and hardware/software optimization, can benefit, as well, by the knowledge of such agendas and goals. Finally, such knowledge can support the design tradeoffs involved in moving cloud services to the edge of the network (so-called fog computing, i.e., defining the right allocation of data storage and processing and offering services).

\section{How Can BPM Benefit From the loT?}

Let us consider a complex process with multiple parties interacting in the context of a business transaction. Such a process can be, for example, procurement, where goods are ordered, delivered, stored, and paid for. While the system can track each automatically executed activity on its own, it relies on messages from other parties and, in the case of manual activities, physically entered information. If these data are not entered, and if they are entered incorrectly, discrepancies between the digital (i.e., computerized representation of the) process and the real-world execution of the procedure occur. Similar concerns hold if the participants do not obey the digital process under certain circumstances, e.g., an emergency in health care, or have not yet entered the data even though the respective activity was already executed through the real-world procedure.

Such scenarios might be better manageable if they closely linked the digital process with the physical world, as enabled by the integration of the IoT and BPM; e.g., the completion of manual activities can be made observable through the use of appropriate sensors (e.g., [6]). The IoT can complete BPM with continuous data sensing and physical actuation for improved decision making. Decisions in processes require relevant information as the basis for making meaningful determinations. In general, it is not sufficient to retrieve these data solely from traditional repositories (e.g., databases and data warehouses); up-todate information is also necessary. Information from the IoT, such as events, that is provided through in-memory 
databases and complex event processing can be useful in this context. The IoT could reduce the need to manually signify the completion of tasks since sensor data would already be available, leading to more accurate information, fewer errors, and efficiency gains.

To provide guidelines for system development, several challenges remain to be tackled. In particular, the following issues must be understood:

- how processes can improve the IoT by 1) taking a process-oriented perspective and considering the process history to 2) bridge the abstraction gap between raw sensor data and higher-level knowledge extracted from that event data and 3) optimize the overall decision making

- how to exploit the IoT for BPM by 1) considering sensor data for automatically detecting the start and end of activities, 2) using event data for making decisions in a predefined process model, and 3) detecting

\section{The Internet of Things}

The Internet of Things (IOT) [5], [S1], [S2] is the internetworking of physical objects (the things), such as embedded systems with electronics hardware, software, sensors, actuators, and network connectivity. Such connected things collect and exchange data. Each thing is uniquely identifiable through its embedded computing system and is able to interoperate within the existing network infrastructure. While things act locally, the IOT enables them to be controlled remotely across existing network infrastructures, including the Internet. The interconnection of these smart objects/things [S3] is expected to usher in automation in nearly all fields. This creates opportunities for more direct integration of the physical world into computer-based and digitized systems and results in improved efficiency, accuracy, and economic benefits as well as increased automation and less human intervention. Experts estimate that the loT will consist of roughly 30 billion objects in 2020 [S4].

\section{References}

[S1] K. Ashton et al., "That 'Internet of Things' thing," RFID J., vol. 22, no. 7, pp. 97-114, 2009.

[S2] J. Holler, V. Tsiatsis, C. Mulligan, S. Avesand, S. Karnouskos, and D. Boyle, From Machine-to-Machine to the Internet of Things: Introduction to a New Age of Intelligence. New York: Academic, 2014.

[S3] G. Kortuem, F. Kawsar, V. Sundramoorthy, and D. Fitton, "Smart objects as building blocks for the Internet of Things," IEEE Internet Comput., vol. 14, no. 1, pp. 44-51, Jan. 2010. doi: 10.1109/ MIC.2009.143.

[S4] A. Nordrum "Popular Internet of Things forecast of 50 billion devices by 2020 is outdated," IEEE Spectrum Tech Talk, Aug. 18, 2016. [Online]. Available: https://spectrum.ieee.org/tech-talk/telecom/ internet/popular-internet-of-things-forecast-of-50-billion-devices -by-2020-is-outdated

\section{Business Process Management}

Business process management (BPM) is a well-established discipline that deals with the identification, discovery, analysis, (re)design, implementation, execution, monitoring, and evolution of organizational procedures [S5]. A business process is a collection of related events, activities, and decisions that involves a number of actors and resources and that leads to an outcome that is of value for an organization or customer. Examples of business processes include order to cash, procure to pay, application to approval, claim to settlement, and fault to resolution.

To support business processes at an operational level, a BPM system (BPMS) can be used. As opposed to data- and function-centered information systems, a BPMS separates process logic from application code and thus provides an additional architectural layer. Typically, a BPMS provides generic services that are necessary for operational, software-enabled business process support, i.e., for process modeling, execution, and monitoring and for user interaction (e.g., worklist management). When using a BPMS, software-enabled business processes are designed in a top-down manner; i.e., process logic is explicitly described in terms of a process model that provides the schema for the process execution. The BPMS is responsible for instantiating new process instances, controlling their execution based on the model, and completing them. The progress of a process instance is typically monitored, and traces of the execution are stored in an event log and can be used for process mining [S6], e.g., the discovery of a process model from the event log and for checking the compliance of the log with a given process model.

So far, the predominant paradigm to develop operational support for business processes has been based on the model-enact paradigm, where the system has been depicted as a (graphical) model that then could be executed by a BPMS. This largely follows a topdown approach and is based on the idea of a central orchestrator that controls the execution of the business process, its data, and its resources. With the emergence of the Internet of Things, the existing model-enact paradigm is challenged by the discover-predict architecture, which can be characterized as a bottom-up approach where data are generated from physical devices that sense their environment and produce raw events. Sensor data then must be aggregated and interpreted to detect activities that can be used as input for process mining algorithms that support decision making.

\section{References}

[S5] M. Dumas, M. La Rosa, J. Mendling, and H. A. Reijers , Fundamentals of Business Process Management, 2nd ed. Berlin: SpringerVerlag, 2018.

[S6] W. Van Der Aalst, Process Mining: Discovery, Conformance and Enhancement of Business Processes, 2nd ed. Berlin: Springer-Verlag, 2016. 
discrepancies between the predefined model and the actual enactment that harnesses event information for online process compliance checking and exception management.

In the remainder of this article, taking these two general questions as starting point, we detail the key points related to combining BPM and the IoT and elaborate on the benefits of BPM for the IoT and vice versa. (This article has its roots in Dagstuhl Seminar 16 191, "Fresh Approaches to Business Process Modeling," organized by Richard Hull, Agnes Koschmider, Hajo A. Reijers, and William Wong at the Leibniz Center for Informatics in Germany, 8-13 May 2016 [25], in which many of the authors participated. Moreover, a preliminary version was published at the Computing Research Repository [26].)

\section{Intersections/Challenges}

The IoT has to deal with a number of challenges; these include, for example, technological barriers, such as the computational limitations of embedded systems and connectivity to back-end systems; security-related concerns; standardization and interoperability matters; data privacy; the untapped potential in data analytics; efficient methods for system organization; and so on [5]. The principal characteristic of the IoT is the communication between loosely coupled objects, which is mostly accomplished asynchronously and ad hoc. BPM deals with the discovery of models, the analysis of predefined models, the adaptation of models, and the enactment of business processes through software applications and systems. Abstract processes can also be discovered from log files, and suitable implementations for instantiation can be predicted.

Accordingly, sensing and perception via sensors and decisions based on sensors as well as determinations based on actuation according to individual goals/strategies constitute fundamental tasks of the IoT. Thereby, sensing constitutes the input and output actuation of any IoT-BPM interaction (see Figure 1). In between, raw event data are processed by event-based systems, transforming the input events to higher-level knowledge. In turn, the latter may be utilized by BPM concepts, methods, and technologies to deal with the discovery of a (process) model, the analysis of a predefined model, the adaptation of a model, and the enactment of a model (a business process). While the IoT generally focuses on communication and data flow, BPM approaches consider control flow, process models (large and "in the large"), and synchronous interactions. In addition, most current BPM approaches have difficulties in dealing with nonroutine, nondeterministic processes, whereas IoT applications typically involve these kinds of interactions. Plenty of intersections, which pose new challenges for researchers and practitioners, arise, as detailed in the following.

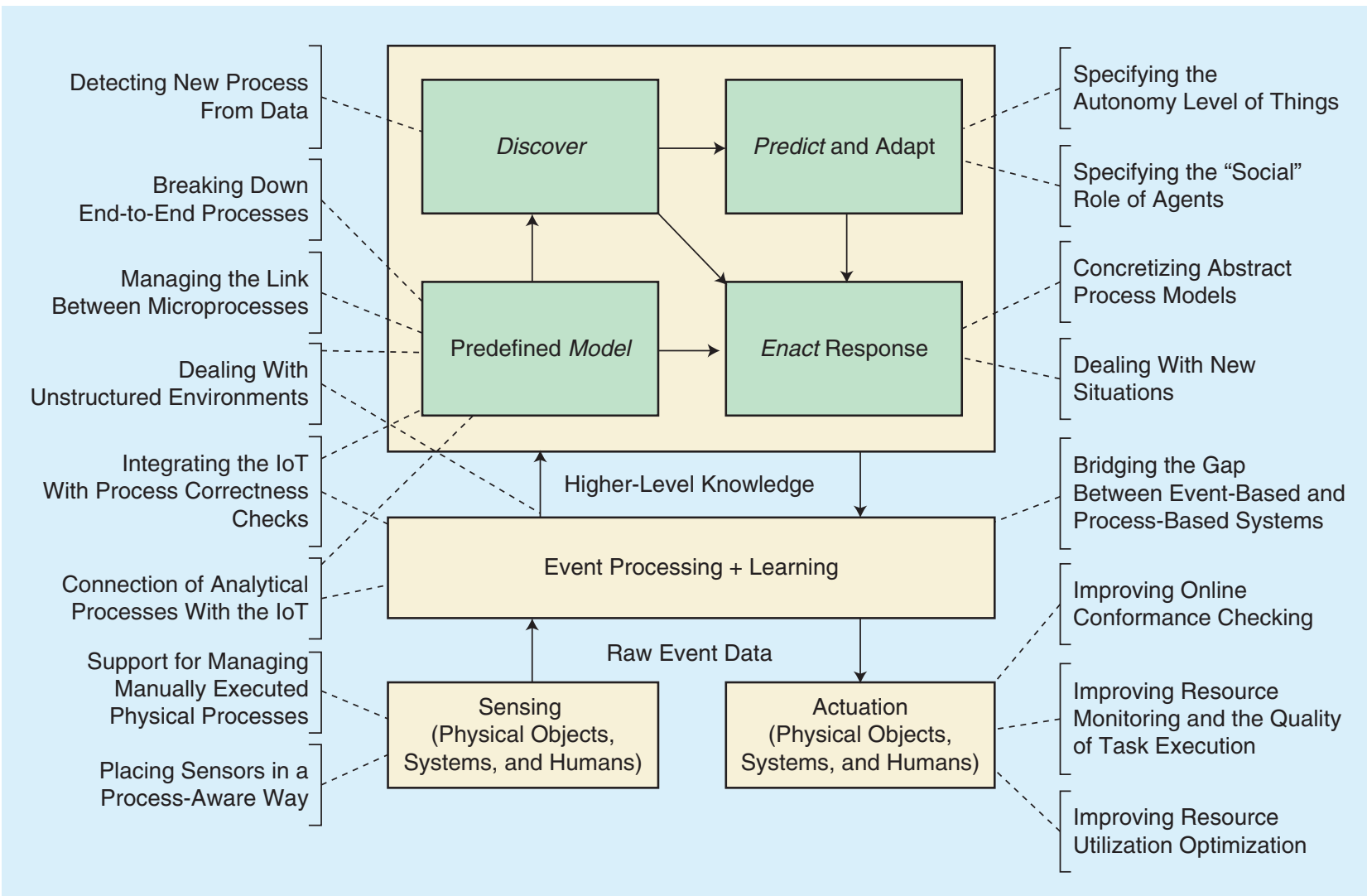

Figure 1. A high-level overview showing the interaction between the loT and BPM. 


\section{Placing Sensors in a Process-Aware Way}

To collect all relevant data, sensors need to be carefully placed. It is already a challenge to construct sensors and locate them on agents (human and artificial) and in smart environments in such a manner that they are nonintrusive and remain efficient. Sensors can be batteryless tags, such as radio-frequency identification tags; powered by batteries, renewable energy, and outlets; and communicate through wired and wireless methods. It is even more challenging to decide on the type of sensor and its placement with regard to its function in respect to the interaction between agents. A (model of a) business process may guide this determination since it offers knowledge about resources, locations, and variants of behavior (enactment) that need to be covered. As well, the tradeoff between the cost of introducing additional sensing points and the expected increase in monitoring accuracy may be approached based on process knowledge.

\section{Support for Managing Manually \\ Executed Physical Processes}

In many scenarios, processes are automated through a BPM system (BPMS), in which some activities require the interplay between human operators and software/hardware modules; in many of these instances, there is an increasing use of mobile devices that foster the delivery of work items to the right users [7]. Workers do not necessarily have to interact with the BPMS while carrying out physical tasks (e.g., moving boxes in a warehouse); sensors, which are connected to the BPMS, monitor whether or not such a chore has started or ended. However, appropriate mappings from process activities to the user interface and usable visualizations are needed to enable actors (process participants) to perform their work in a natural way, without requiring nonvalue-adding management duties, such as clicking on confirmation buttons.

\section{Connection of Analytical Processes With the loT}

During process execution, a variety of information is required to make meaningful decisions. In turn, this information often needs to be available from traditional databases/data warehouses of historical data, and it must also be current. It needs to be clear where data come from and where they have been used (data provenance), and overall quality requirements for the information must be ensured. It becomes necessary to find a way to annotate the origin of data and use this (meta)information in process models. So far, there is no widely accepted approach to connect the analytic processes of observation, analysis, and decision making to business processes in a standardized way; recent attempts include the decision model and notation standard. Its focus, however, is on decision requirements and less on the origin and use of decision data. Hence, a technique is still needed to model data quality and provenance so that information can be exploitable at the process model level. Erroneous sensors, which do not work at all or deliver erroneous data, must be discovered and excluded from any analysis. In turn, a reasonable judgment about which sensor data might be erroneous is required. The process context in which these data occur might be helpful to identify and cope with erroneous sensors.

\section{Integrating the IoT With Process Correctness Checks}

Well-known techniques for analyzing process models can contribute to improve the design of interactions in the IoT by finding deadlocks, livelocks, and dead activities in interactions between smart objects. Deadlocks and livelocks are reasons why some processes may not terminate in the assumed time frame or at all. While a rollback is a typical service in data management, it becomes much costlier and more complicated when managing processes and thus should be avoided. Dead activities do not harm processes execution (unless they are supposed to be mandatory) since they will never be triggered. Yet, they represent a waste of resources, as physical and virtual elements may have been reserved for these activities. Therefore, designing correct process models that specifically consider the IoT nature of some components becomes crucial, as does the verification of important properties.

\section{Dealing With Unstructured Environments}

BPM offers a way to structure businesses. Thus, it often assumes a controlled environment with a managed repository of versioned processes that can be orchestrated for the purpose of a single enterprise and be choreographed between parties in cases of cross-organizational collaborations. Orchestration denominates the execution order of the interactions from the perspective, and under the control, of a single party, whereas choreography describes public, i.e., globally visible, message exchanges, interaction rules, and agreements made among multiple entities. Both concepts presume knowledge about the structure and interactions of each participating process. It is questionable whether orchestration and choreography still suffice as organizational concepts in an IoT world, which is much more ad hoc and situational (e.g., devices involved in the interaction might fail and deliver erroneous data, new devices may have to be added flexibly, and so forth).

\section{Managing the Links Between Microprocesses}

One approach to bridge the gap between IoT data and processes would be to break end-to-end models into microprocesses that represent habits and arrange them in a less prescriptive (control flow) way. Modeling a small and possibly autonomous microprocess does not necessarily require new modeling constructs and methods. Yet, the organization of hundreds or thousands of loosely coupled small processes may require new modeling constructs and methods to structure and represent nonhierarchical interactions in a human-readable form. Data-centric process paradigms offer promising perspectives in this context [8]. 
For example, object-aware processes describe the behavior of single objects through microprocesses, whereas the dynamic construction of linked objects and their synchronized execution is described and enforced through macroprocesses. However, the respective approaches need to be enhanced to integrate physical objects as well as their behavior into the overall process.

\section{Breaking Down End-to-End Processes}

For a large class of processes (typically referred to as dynamic or knowledge intensive [9]), the advent of an overwhelming amount of sensor data and a tremendous number of things (which act in an environment where they encounter no central control and behave according to "personal" agendas) makes it practically impossible to define comprehensive end-to-end process models. Things will perform their own routines, so-called repeated behavior patterns, or habits (possibly to be mined; see [10]). Accordingly, processes will have to be organized as event-driven microprocesses to represent these habits. Whereas the end-to-end business process itself may be modeled in traditional ways, the linking of microprocess models is far more complex; to cope with this emerging intricacy, the possible interactions between microprocess models must not be described at the low level of message exchanges but through a higher semantical level, similar to the utilization of semantic object relations for the purpose of object interactions in objectaware process management. Figure 2 gives the intuition of such a complex interplay.

\section{Detecting New Processes From Data}

Designing a system in a bottom-up manner without prescriptive process models promises more flexible and inclusive procedures. However, the question arises: To what extent we can let the system just evolve and be discovered? When developing support for software-enabled business processes based on the principles of the IoT, an evolutionary self-organizing process will take place in some respect. Thus, one must find the appropriate level of structuring and prescription without harming the capability to self-organize. There is a gap between IoT data and concepts at a model level to enable behavior prediction and identify changes in behavior. The IoT

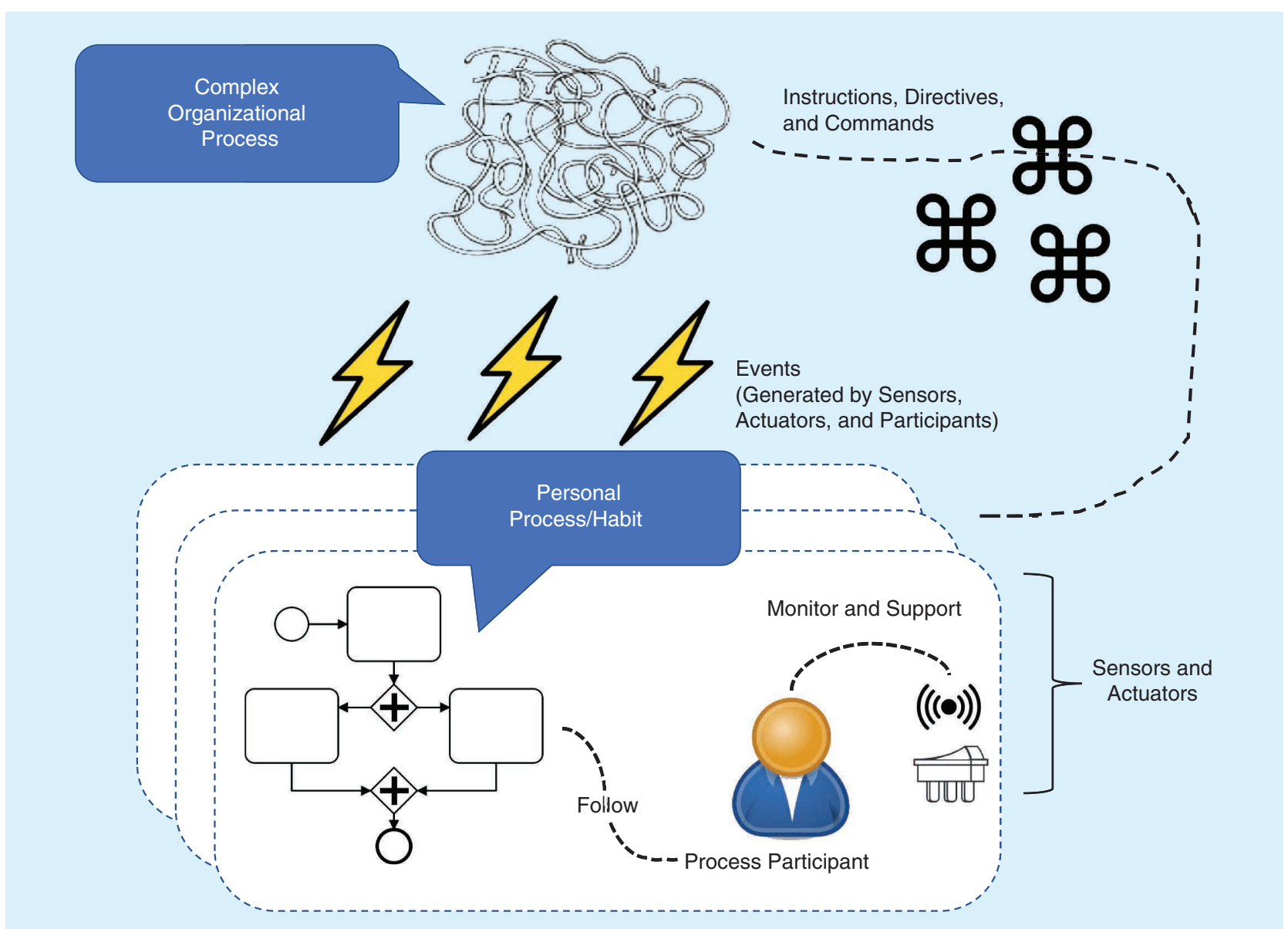

Figure 2. The interplay of personal processes/habits with respect to complex organizational processes. Process participants follow habits and are monitored and supported by sensors and actuators. Events and instructions/ directives/commands interconnect the two layers but without any rigid prescription and possibly through models that are to be dynamically mined. 
facilitates deriving situational knowledge when tracking and evaluating data streams. Situational knowledge, in turn, is input to analyze prospective knowledge, which constitutes a dynamic task. Prospective knowledge addresses long-tail information about resources (How well is the person/thing doing? Are there any behavior changes expected?). Moreover, data streams from sensors need to be tracked, mapped to information entities, and simulated. Additionally, the output (goal) must be known (e.g., save time, save costs, and improve health), and its derivation and the reconciliation of private goals must be mapped with organizational targets, which is a challenge of the IoT. An alignment between event-based and process-oriented systems is indispensable in this context. A starting point could be to define goal-based deviation patterns and provide modeling techniques that consider sensor and event data.

\section{Specifying the Autonomy Level of Things}

Objects in the IoT are able to react to events by executing tasks and entire processes. The execution of the latter procedures is typically asynchronous and sometimes not explicitly begun from a central coordinator. The execution of tasks and processes may further trigger certain reactions, for example, the start of another process to correct deviating behavior. Yet, it is unfeasible to grant things full autonomy to decide everything without supervision. Hence, there has to be a concept of autonomy levels that dictates whether things, be they individuals or in a group, need supervision and whether the things' decisions may be vetoed. Currently, there is no universal way to represent these levels of autonomy and resolve conflicts originating from this distinction [11], [12]. While different conceptualizations of individual and group autonomy exist, they have not yet been transferred to BPM and the IoT.

\section{Specifying the "Social" Role of Agents}

Institutions aim to optimize their business processes based on organizational (i.e., group) goals. However, process participants often follow personal, i.e., individual, processes and agendas. The challenge is to synchronize/ reconcile different, possibly conflicting goals. These agendas are typically mitigated through governance processes that prescribe desired behaviors. The individual goals of a thing are typically not precisely known and explicitly given. Furthermore, these processes may be less prescriptive microprocesses and habits. Hence, holistic and prescriptive governance may not be possible. Thus, it is an option to define and specify the "social" behavior of things (such as self-interest, helping, and being cooperative [13]) to better coordinate and govern their behaviors. This becomes even more challenging with the integration of human actors as well as robots in processes (raising issues such as exchangeability, the coexistence of different kinds of resources, and so on).

\section{Concretizing Abstract Process Models}

Abstract models are sometimes used to replicate processes at design time without providing the details that are necessary for execution. This is a sensible approach when dealing with very dynamic scenarios. In these cases, it is possible to define the process, but the abstract model has to be turned into a concrete version before it is executable, for example, by discovering available services as well as the conditions in which the services may be used. The context also includes physical data about users, e.g., location and the devices a user carries with him or her (such as smartphones). For the discovery phase, the semantics related to the services (i.e., what functionality the service can offer, especially within the context of the process) should be available, and it should be possible to reason through this for matchmaking purposes. In addition, the services' discovery phase may lead to changes in the schema of the original abstract process. Examples of corresponding changes include the skipping of certain tasks that were initially planned for a process and the addition of new fragments (e.g., combining two or more services either in sequence or in parallel to achieve the task goal).

\section{Dealing With New Situations}

Individual ad hoc decisions may favorably resolve a current situation from an individual's or a small group's point of view. In a complex business environment, decision making conducted with foresight and structure achieves similar results and saves money and time, possibly improving overall quality. Deterministic event detection and correlation can be very well modeled and executed with event processing languages in complex event processing engines. However, the flexible discovery of new situations and the derivation of original responses constitute major technological challenges whose resolutions can benefit from the combination with BPM.

BPM methodologies and technologies can support the identification and selection of appropriate responses by recommending tasks, triggering tasks and whole processes, and automating as well as monitoring task execution. These reactions can be predefined using existing BPM technologies, and learning can be based on the analysis of historic traces to identify beneficial habits from a higherlevel perspective. Furthermore, reference models [14] can help identify state-of-the-art industry blueprints, which can be contextualized and instantiated to find a proper reaction for the context and the history of the situation. The capability of IoT sensing can be of additional benefit here.

\section{Bridging the Gap Between Event-Based and Process-Based Systems}

A challenge is to bridge the gap between clouds of sensor data and event logs for process mining. Events captured by sensors are available in high volumes, velocities, and varieties. They are often affected by noise and errors. Process knowledge can be employed to support the identification 
of events from raw event data and, in a subsequent step, entire processes and their activities from event data. This is a nontrivial problem since event data belonging to different activities can be interleaving. Moreover, event information can belong to, or be relevant for, several activities so that complex N:M relations between events and activities have to be considered.

Once the activities have been identified, the next challenge is to discover the corresponding processes, i.e., to correlate the activities with the corresponding process instances. Process knowledge and BPM methodologies (e.g., [15]) can support the discovery and identification of underlying interactions as processes as well as the optimization to reduce wasted time and resources and increase the safety of all involved agents. Process mining techniques provide promising ex post perspectives in this respect but require the presence of a log that organizes the events in terms of traces representing the execution of a process instance. Similarly, but in an online fashion, complex event processing can be used to derive higher-level knowledge from raw events to provide an ex nunc perspective [2]. Here, the timely provisioning of events is crucial.

\section{Improving Online Conformance Checking}

Conformance checking is a process mining technique that compares an existing process model with an event log of the same process. It can be used to check if the reality of process execution, as recorded in the log, conforms to the model and vice versa. Online conformance checking takes as input the context information and performs the comparison. This requires high-quality data and almost complete information. Again, the IoT, as a data source, and data management technologies can play a major role and might improve the conformance checking of the actual physical execution with the execution order as recorded by the BPMS based on a secondary log of sensor information. Similarly, IoT data can be used for checking and monitoring compliance rules that must be obeyed during process execution.

\section{Improving Resource Utilization Optimization}

BPM can provide a governance structure for physical and virtual organizations. BPM initiatives break up traditional functional silos and introduce process managers to being responsible for processes across departments. While complex systems and the IoT are centered around situations that they must react to, BPM initiatives are organized around processes. This ensures that some coordination instance that is responsible for priorities and resource provisioning can monitor events and intervene with additional knowledge, if necessary. In a pure IoT paradigm, there is a danger that decisions will produce only local optima. The coordinating unit responsible for resource provisioning has advanced knowledge about the future behavior of agents since the agents have to follow their process models and thus can provide resources (e.g., computing power, network bandwidth, and things) with greater accuracy, reducing the processing time and increasing the throughput. This also helps to reduce communication time-outs and rollbacks as well as abnormal process terminations (see some initial results in [16] and [17]).

\section{Improving Resource Monitoring and the Quality of Task Execution}

The execution of tasks in a business process consumes resources, including IT, such as the storage capacity for processed data; computing power for calculations in scientific workflows; artificial agents, such as robots that automatically execute manual tasks; and human beings who enter and analyze data and perform manual tasks. Also, machines, e.g., those for packing drugs, can be considered as resources (e.g., predictive monitoring; i.e., when the machine has to be maintained, taking its usage and historical data into account).

All these resources might suffer from issues that hinder optimal working conditions, such as over- and underutilization and even damage/illness. IoT-based sensors can pick up these issues by measuring machine behaviors and human stress levels [18] and suggest changes to the process execution to alleviate the effects. Furthermore, the IoT can support the execution of (knowledge-intensive) tasks in a process through context-specific knowledge provisioning, e.g., in terms of instructions and training materials covering how to execute a task and regulations that are relevant to a user's particular context. Sensor data can be leveraged to determine the actual context and identify information needs (e.g., the detection of cognitive overload and stress).

\section{Concluding Remarks}

The IoT provides many opportunities for organizations/ companies/industries and for personal use through the meaningful yet dynamic interaction of humans, software, machines, and things. BPM is a well-established discipline that deals with the discovery, analysis, (re)design, implementation, execution, monitoring, control, and evolution of business processes. So far, both areas have been separately considered. In this article, we formulated a number of points for the amalgamation of the IoT and BPM, which we deem important to be tackled in the near future for the IoT to benefit from business processes and vice versa.

When adopting the IoT and BPM in the building of complex systems, we need to carefully consider specific application scenarios; therefore, the generalizability and adoption of practices, patterns, and modeling approaches may be questionable. One of the challenging concepts presented in this article is that general modeling, design, and mining approaches should be devised to enable the consideration of different applications. An interesting preliminary question is how to classify IoT applications, and according to which dimensions, to perform such a 
generalization (see an initial study in [19]). Also, not all scenarios for potential IoT applications can equally benefit from BPM; e.g., the single app-controlled Phillips Hue lamp will not profit from BPM concepts, whereas a scenario that schedules maintenance appointments for a fleet of cars might.

Before concluding, we would like to highlight a crossissue, i.e., dealing with security and, in particular, privacy issues. Privacy levels that exist at the sensor layer might be different with respect to those at the BPM one. A fulldisclosure approach should be avoided, especially in contexts where sensitive (i.e., personal) information is collected. The most relevant challenge, in this case, is the communication between the two worlds, each of them with corresponding privacy/security levels and policies. The layer in charge of integrating these two sides should be planned according to the principles of privacy by design [20]: "Identify and examine possible data protection problems when designing new technology and to incorporate privacy protection into the overall design, instead of having to come up with laborious and time-consuming "patches' later on" [21]. This issue can also be seen as a "nonfunctional requirement," referring to the points discussed in the "Intersections/Challenges" section, but others might also be affected.

Finally, and partially related to the previous point, there are ethical aspects of integrating the IoT and BPM: the introduction of raw events paves the way for a whole new set of analyses and explorations. On the one hand, these analyses must preserve the privacy of the individual (privacy is recognized as a fundamental right; see [27] and [28]). At the same time, the analyses should not be unfair and should not provide unequal treatment of people based on membership to a category or a minority. This problem is typically referred to as discrimination-aware data mining [22]. More generally, the literature also talks about "privacy-preserving data mining" [23]. There are several points that are directly affected, such as those in the "Intersections/Challenges" section, due to the set of analyses that the integration of the IoT and BPM will make possible.

\section{About the Authors}

Christian Janiesch (christian.janiesch@uni-wuerzburg .de) is an assistant professor at the University of Würzburg. He is a member of the Business \& Information Systems Engineering department editorial board and has authored more than 100 scholarly publications. His research interests lie at the intersection of business process management and business analytics, with frequent applications in the Industrial Internet of Things.

Agnes Koschmider (ak@informatik.uni-kiel.de) is a professor of information systems at Kiel University and head of the Process Analytics group. Her work has been published in more than 90 research papers and articles. Her research interests broadly concern how to extract meaning- ful event logs from Internet of Things (information) systems, with a special focus on privacy preservation.

Massimo Mecella (massimo.mecella@uniroma1.it) is an associate professor at Sapienza Università di Roma. His research interests focus on business process management, cyberphysical systems and the Internet of Things, advanced interfaces and human-computer interaction, and software architectures and service-oriented computing, with applications in multiple fields, including digital government, smart spaces, Industry 4.0, health care, disaster/crisis response and management, cybersecurity, and digital humanities.

BarbaraWeber (barbara.weber@unisg.ch) earned her Ph.D. degree in business administration from the University of Innsbruck, Austria. She is chair of software systems programming and development at, and director of, the Institute of Computer Science at the University of St. Gallen, Switzerland. Her research interests include the development of adaptive software systems, including flexible and adaptive business process support and neuroadaptive information systems as well as human and cognitive aspects of software and information systems engineering.

AndreaBurattin (andbur@dtu.dk) earned his Ph.D. degree from the Universities of Bologna and Padua (Italy). He is an associate professor at the Technical University of Denmark and, since 2019, has been a member of the IEEE Task Force on Process Mining Steering Committee. The IEEE Task Force on Process Mining awarded his doctoral thesis the Best Process Mining Dissertation Award for dissertations defended in 2012-2013.

Claudio Di Ciccio (claudio.diciccio@uniroma1.it) is an assistant professor at Sapienza Università di Roma, Italy. He was program committee (PC) chair for the first Blockchain Forum at the International Conference on Business Process Management 2019 and regularly serves on the PCs of the conferences in the area. He is a member of the IEEE Task Force on Process Mining Steering Committee. His research interests include process mining, declarative modeling, and blockchains.

Giancarlo Fortino (giancarlo.fortino@unical.it) is a full professor at the University of Calabria (Unical), Italy. He is director of the Smart, Pervasive, and Mobile Systems Engineering lab at Unical as well as cochair of joint labs to study the Internet of Things (IoT) that were established between Unical and Wuhan University of Technology, Southern Medical University, and Huazhong Agricultural University in China. In addition, he is cofounder and chief executive officer of SenSysCal, a Unical spinoff focused on innovative IoT systems. His research interests include agent-based computing, wireless (body) sensor networks, and the IoT.

Avigdor Gal (avigal@ie.technion.ac.il) is a full professor of data science at Technion-Israel Institute of Technology. He has served as a program and general chair of the International Conference on Business Process Management and the International Conference on Distributed EventBased Systems. He is the author of Uncertain Schema Matching. His research interests include data integration, 
business process management, cyberphysical systems, and the Internet of Things (IoT), with applications in multiple fields, including smart cities and the food IoT.

Udo Kannengiesser (udo.kannengiesser@jku.at) is a professor at Johannes Kepler University Linz, Austria. He previously worked for several software companies, research institutes, and universities in Australia, Germany, and Austria. His research interests include design computing, design cognition, design creativity, design methodology, distributed systems, process management, and digital manufacturing.

Francesco Leotta (francesco.leotta@uniroma1.it) is an assistant professor in the Department of Computer, Control, and Management Engineering, Sapienza Università di Roma, Italy. His research interests concern algorithmic, methodological, experimental, and practical aspects in different areas of computer science, including ubiquitous computing, human-computer interaction, and digital humanities. Such topics are challenged in the application domains of smart spaces, smart manufacturing, and cultural heritage.

Felix Mannhardt (felix.mannhardt@sintef.no) is a parttime associate professor at NTNU Norwegian University of Science and Technology, Trondheim, Norway. He also works as research scientist in the technology management department of SINTEF Digital, where he co-founded the company KIT-AR. He is a member of the IEEE Task Force on Process Mining and contributed significantly to the ProM and bupaR frameworks. His research interests include using low-level event data for process mining as well trust and privacy concerns connected to process mining.

Andrea Marrella (andrea.marrella@uniroma1.it) is an assistant professor at Sapienza Università di Roma, Italy. Since 2017, he has been information director of Journal of Data and Information Quality. His research interests focus on how to integrate artificial intelligence with business process management solutions to untangle complex challenges from the fields of process mining and robotic process automation.

Jan Mendling (jan.mendling@wu.ac.at) is a full professor at Wirtschaftsuniversität Wien, Vienna, Austria. He is a member of the board of the Austrian Society for Process Management (http://prozesse.at), a cofounder of the Berlin Business Process Management (BPM) Community of Practice (http://www.bpmb.de), and a member of the IEEE Task Force on Process Mining. He is a coauthor of the textbooks Fundamentals of Business Process Management (http://fundamentals-of-bpm.org/) and Wirtschaftsinformatik. His research interests include BPM and information systems.

Andreas Oberweis (andreas.oberweis@kit.edu) has been a professor of applied informatics at the Karlsruhe Institute of Technology (KIT) since 2003. He is a board member of the FZI Research Center for Information Technology in Karlsruhe and a cofounder of several companies in the field of business process management. His research interests lie at the border between soft- ware engineering, business process management, and database management.

Manfred Reichert (manfred.reichert@uni-ulm.de) is a professor of computer science at, and director of, the Databases and Information Systems Institute, Ulm University, Germany. He was a cofounder of AristaFlow, and several of his techniques have been applied in health care, logistics, automotive engineering, and Industry 4.0. His research interests span the fields of process and data science, information systems engineering, and digital services.

Stefanie Rinderle-Ma (stefanie.rinderle-ma@univie ac.at) is a full professor in the Faculty of Computer Science, University of Vienna, Austria. Her research interests include process-aware information systems, distributed process technology, digitalized compliance management, and business process intelligence, with application areas such as manufacturing and health care.

Estefanía Serral (estefania.serralasensio@kuleuven .be) is an assistant professor at KU Leuven, Belgium. In 2018, she was an assistant professor at Eindhoven University of Technology, The Netherlands. From 2012 to 2014, she led the Semantic Knowledge Representation and Integration research group at the Christian Doppler Laboratories, Technical University of Vienna, Austria. She has authored more than 100 research papers in conferences and journals. Her research interests focus on the IoT, business process management, and context-adaptive systems.

WenZhan Song (wsong@uga.edu) is a chair professor of electrical and computer engineering at the University of Georgia. His research interests include cyberphysical systems informatics and security and their applications in energy, health, and environment systems, where distributed sensing, networking, computing, and security play a critical role and need a transformative study.

Jianwen Su (su@cs.ucsb.edu) is a professor of computer science at the University of California, Santa Barbara. He has chaired different conferences, including the Symposium of Principals of Database Systems, Association for Computing Machinery Special Interest Group on Management of Data Conference, and the International Conference on Service-Oriented Computing. His research interests include data management and business process management, the Internet of Things, and service-oriented computing.

Victoria Torres (vtorres@pros.upv.es) is an associate professor at Universitat Politècnica de València. She is a member of the Valencian Research Institute for Artificial Intelligence, working actively in areas such as business process management, the Internet of Things, metamodeling, web engineering, and model-driven development. Since 2010, she has participated in national and European projects (Open Platform for the Engineering of Embedded Systems, State Information and Technology Advisory Committee, and Developer Companion for Documented and Annotated Code Reference).

Matthias Weidlich (matthias.weidlich@hu-berlin.de) is a professor and chair of databases and information systems 
in the Department of Computer Science, Humboldt-Universität zu Berlin. He serves as editor-in-chief of Information Systems and is a member of the steering committee of the Association for Computing Machinery International Conference on Distributed Event-Based Systems. His research interests focus on process-oriented and event-based information systems.

Mathias Weske (mathias.weske@hpi.de) is a professor and chair of the Business Process Technology research group at the Hasso Plattner Institute, Digital Engineering Faculty, University of Potsdam, Germany. He is a founding member of the steering committee (of which he has been chair since September 2017) of the International Conference on Business Process Management. He is on the editorial board of Computing. His research interests include the engineering of processoriented information systems, process mining, and event processing.

Liang Zhang (lzhang@fudan.edu.cn) is a full professor of computer science at Fudan University, China. His current research interests include Internet of Thingsenabled information systems and reactive systems, mainly in the form of service-based and business process-oriented ones.

\section{References}

[1] L. Jiang, L. D. Xu, H. Cai, Z. Jiang, F. Bu, and B. Xu, "An IoT-oriented data storage framework in cloud computing platform," IEEE Trans. Ind. Informat., vol. 10, no. 2, pp. 1443-1451, 2014. doi: 10.1109/TII.2014.2306384.

[2] C. Batini and M. Scannapieco, Data and Information Quality. Cham, Switzerland: Springer-Verlag, 2016.

[3] C. Janiesch, M. Matzner, and 0. Müller, "Beyond process monitoring: A proof-ofconcept of event-driven business activity management," Bus. Process Manage. J., vol. 18, no. 4, pp. 625-643, 2012. doi: 10.1108/14637151211253765.

[4] A. Marrella, M. Mecella, and S. Sardina, "Supporting adaptiveness of cyber-physical processes through action-based formalisms," AI Commun., vol. 31, no. 1, pp. 47-74, 2018. doi: 10.3233/AIC-170748.

[5] J. Gubbi, R. Buyya, S. Marusic, and M. Palaniswami, "Internet of Things (IoT): A vision, architectural elements, and future directions," Future Gener. Comput. Syst., vol. 29, no. 7, pp. 1645-1660, Sept. 2013. doi: 10.1016/j.future.2013.01.010.

[6] F. Stertz, J. Mangler, and S. Rinderle-Ma, "NFC-based task enactment for automatic documentation of treatment processes," in Proc. Int. Workshop Business Process Modeling, Development and Support, Int. Conf. Evaluation and Modeling Methods of Systems Analysis and Development, 2017, pp. 34-48. doi: 10.1007/978-3-319-59466-8_3.

[7] R. Pryss and M. Reichert, "Robust execution of mobile activities in process-aware information systems," Int. J. Inform. Syst. Model, vol. 7, no. 4, pp. 50-82, 2016. doi: 10.4018/IJISMD.2016100104.

[8] S. Steinau, A. Marrella, K. Andrews, F. Leotta, M. Mecella, and M. Reichert, "DALEC: A framework for the systematic evaluation of data-centric approaches to process management software," Softw. Syst. Model., vol. 18, no. 4, pp. 1-38, 2019. doi: 10.1007/s10270-018-0695-0.

[9] C. Di Ciccio, A. Marrella, and A. Russo, "Knowledge-intensive processes: Characteristics, requirements and analysis of contemporary approaches," J. Data Semant., vol. 4, pp. 29-57, Mar. 2015. doi: 10.1007/s13740-014-0038-4.

[10] F. Leotta, M. Mecella, and D. Sora, "Visual process maps: A visualization tool for discovering habits in smart homes," J. Ambient Intell. Human. Comput., vol. 11, no. 5, pp. 1997-2025, 2020. doi: 10.1007/s12652-019-01211-7.
[11] R. Parasuraman, T. B. Sheridan, and C. D. Wickens, "A model for types and levels of human interaction with automation," IEEE Trans. Syst., Man, Cybern. A: Syst. Humans, vol. 30, no. 3, pp. 286-297, 2000. doi: 10.1109/3468. 844354.

[12] M. Schillo and K. Fischer, "A taxonomy of autonomy in multiagent organisation," in Proc. Int. Workshop Computational Autonomy, 2003, pp. 68-82. doi: 10.1007/978-3540-25928-2_6.

[13] S. Kalenka and N. R. Jennings, "Socially responsible decision making by autonomous agents," in Cognition, Agency and Rationality. Berlin: Springer-Verlag, 1999, pp. $135-149$.

[14] P. Fettke and P. Loos, Reference Modeling for Business Systems Analysis. Idea Group, Hershey, PA, 2007.

[15] A. Senderovich, A. Rogge-Solti, A. Gal, J. Mendling, and A. Mandelbaum, "The ROAD from sensor data to process instances via interaction mining," in Proc. Int. Conf. Advanced Information Systems Engineering (CAiSE 2016), Ljubljana, Slovenia, June 13-17, 2016, pp. 257-273. doi: 10.1007/978-3-319-39696-5_16.

[16] C. Janiesch, I. Weber, J. Kuhlenkamp, and M. Menzel, "Optimizing the performance of automated business processes executed on virtualized infrastructure," in Proc. 2014 47th Hawaii Int. Conf. System Sciences, pp. 3818-3826. doi: 10.1109/ HICSS.2014.474.

[17] S. Schulte, C. Janiesch, S. Venugopal, I. Weber, and P. Hoenisch, "Elastic business process management: State of the art and open challenges for BPM in the cloud," Future Gener. Comput. Syst., vol. 46, pp. 36-50, May 2015. doi: 10.1016/j. future.2014.09.005.

[18] M. T. Adam, H. Gimpel, A. Maedche, and R. Riedl, "Design blueprint for stress-sensitive adaptive enterprise systems," Bus. Inform. Syst. Eng., vol. 59, no. 4, pp. 277-291, 2017. doi: 10.1007/s12599-016-0451-3.

[19] S. Mandal, M. Hewelt, M. Oestreich, and M. Weske, "A classification framework for IoT scenarios," in Business Process Management Workshops: BPM 2018 International Workshops, Sydney, NSW, Australia, September 9-14, 2018, Revised Papers (Lecture Notes in Business Information Processing), vol. 342, Berlin: Springer-Verlag, 2018, pp. $458-469$.

[20] M. Langheinrich, "Privacy by design-Principles of privacy-aware ubiquitous systems," in Proc. Int. Conf. Ubiquitous Computing, 2001, pp. 273-291. doi: 10.1007/3540-45427-6_23.

[21] P. Schaar, "Privacy by design," Identity Inform. Soc., vol. 3, no. 2, pp. 267-274, 2010. doi: 10.1007/s12394-010-0055-x.

[22] D. Pedreshi, S. Ruggieri, and F. Turini, "Discrimination-aware data mining," in Proc. 14th ACM SIGKDD Int. Conf. Knowledge Discovery and Data Mining, 2008, pp. 560-568. doi 10.1145/1401890.1401959.

[23] J. Vaidya, C. W. Clifton, and Y. M. Zhu, Privacy Preserving Data Mining, vol. 19, Berlin: Springer-Verlag, 2006.

[24] International Telecommunication Union. "Internet of things global standards initiative." Accessed: Aug. 18, 2020. [Online]. Available: https://www.itu.int/en/ITU-T/ gsi/iot/Pages/default.aspx

[25] R. Hull, A. Koschmider, H. A. Reijers, and W. Wong. "Fresh approaches to business process modeling." Dagstuhl Research Online Publication Server. http://drops.dagstuhl .de/opus/volltexte/2016/6696/ (accessed Aug. 18, 2020).

[26] C. Janiesch et al., "The Internet of Things meets business process management: Mutual benefits and challenges," 2017, arXiv:1709.03628.

[27] European Court of Human Rights. "European convention on human rights." Accessed: Aug. 18, 2020. [0nline]. Available: http://www.echr.coe.int/Documents/ Convention_ENG.pdf

[28] United Nations Human Rights. "Universal declaration of human rights." Accessed: Aug. 18, 2020. [Online]. Available: https://www.ohchr.org/EN/UDHR/Documents/ UDHR_Translations/eng.pdf 